\title{
Combinatorial aspects of pyramids of one-dimensional pieces of fixed integer length
}

\author{
Bergfinnur Durhuus $\rrbracket$ and Søren Eilers $\|^{\prime}$ \\ ${ }^{1}$ Department of Mathematical Sciences, University of Copenhagen, Universitetsparken 5, DK-2100 Copenhagen Ø
}

\begin{abstract}
We consider pyramids made of one-dimensional pieces of fixed integer length $a$ and which may have pairwise overlaps of integer length from 1 to $a$. We give a combinatorial proof that the number of pyramids of size $m$, i.e., consisting of $m$ pieces, equals $\left(\begin{array}{c}a m-1 \\ m-1\end{array}\right)$ for each $a \geq 2$. This generalises a well known result for $a=2$. A bijective correspondence between so-called right (or left) pyramids and $a$-ary trees is pointed out, and it is shown that asymptotically the average width of pyramids equals $\sqrt{\frac{\pi}{2} a(a-1) m}$.
\end{abstract}

Keywords: heaps, pyramids, polyominoes, lattice animals, enumeration, trees, Dyck paths, LEGOs

\section{Introduction}

Solutions of the enumeration problem for a variety of lattice animals have been obtained in recent years, although the problem concerning general animals on the square or the triangular lattice both remain unsolved. The problems considered in this area are interesting in their own right from a combinatorial point of view, some of them being equivalent to other well known combinatorial problems, and frequently they are inspired by concrete problems in other fields. Thus, for the standard model of site percolation on a lattice [13], the connected percolation clusters are lattice animals and their combinatorial properties of imminent importance for the critical properties of the model [9]. Viewing the points in a lattice as centers of the elementary cells of the dual lattice, an animal can be identified with an edge-connected set of elementary cells on the dual lattice, also called a polyomino. Thus, enumerating lattice animals and polyominoes amounts to the same problem, although the motivation for studying a certain class of animals and its polyomino counterpart may be quite different. Early enumeration results for polyominoes can be found in [16, 23]. For more recent results see [6, 26] and references given there.

A particular, much studied, problem is that of directed animals on a square lattice, first solved by Dhar in [10], and later by a number of authors using different methods [15, 14, 19, 4, 5, 3, 20, 6]. A directed animal on the square lattice is a set of points on the lattice such that any point in the set is the end point of a lattice path starting at the origin all of whose steps are directed towards either east or north and all of whose points are contained in the set. The perhaps most elegant solution to this problem [6] is obtained

\footnotetext{
†durhuus@math.ku.dk

‡eilers@math.ku.dk

1365-8050 @ 2010 Discrete Mathematics and Theoretical Computer Science (DMTCS), Nancy, France
} 
by observing [24] that if the lattice is rotated through an angle $\pi / 2$ counterclockwise and each point in a directed animal is replaced by a suitable dimer, one obtains a pyramid of dimers, the detailed definition of which is given below, restricted such that no dimer is placed directly on top of another. The generating function for such pyramids can then be obtained rather simply as a solution of an algebraic equation. In turn, the solution to the directed animal problem on the triangular lattice can be obtained using that those animals correspond to general pyramids of dimers [24].

In this paper we address the problem of enumerating pyramids whose pieces are of fixed but arbitrary integer length $a$ instead of dimers (which correspond to $a=2$ ). These may, of course, be viewed as a particular type of polyominoes on the square lattice, or one may think of them as connected, planar LEGOs made of $1 \times a$-pieces and which are obtained by dropping successively pieces from above so that the resulting configuration is connected.

More precisely, we shall consider heaps in the sense of Viennot [25] whose basic pieces are onedimensional and have fixed integer length $a$ and whose concurrency relation is defined by assuming each piece to be an interval ] $s, s+a[, s \in \mathbb{Z}$, and two intervals $\alpha, \beta$ are concurrent if and only if $\alpha \cap \beta \neq \emptyset$. Thus a heap, in this article, can be thought of as being obtained by dropping a finite number of pieces towards a horizontal axis. Recall that a heap is a pyramid if it has a unique bottom piece. We call a pyramid $p$ a right s-pyramid, if the bottom piece covers the interval $] s, s+a[$ and is a leftmost piece in $p$. Similarly, $p$ is a left s-pyramid if the bottom piece covers the interval $] s-a, s[$ and is a rightmost piece in $p$.

When using the term pyramid it will henceforth be assumed, unless otherwise stated, that its bottom piece covers the interval $] 0, a[$. The number of pieces in a pyramid $p$ will be called its size and is denoted by $|p|$. Moreover, the width of a pyramid is defined as the length of its projection onto the horizontal axis.

We then have

Theorem 1.1 Given $a \geq 2$, the number of pyramids of size $m$ equals $\left(\begin{array}{c}a m-1 \\ m-1\end{array}\right)$.

This result is well known for $a=2$ [6]. For $a>2$ a proof can be based on the abstract theory of heaps, in particular Proposition 6.3 of [25] (however, a proof of the proposition is not provided in [25]). We give in this note an elementary combinatorial proof using a method that allows addressing various other combinatorial properties of pyramids besides enumeration. The case $a=2$ is reconsidered in Section 2 for the purpose of illustrating our strategy which, in particular, involves establishing a bijective correspondence between pyramids of size $m$ and closed walks on the integers of length $m$. In Sections 3 and 4 we generalise in two steps this correspondence to the case $a \geq 3$ from which Theorem 1.1 will follow. Although our method has common features with that of [6] it is worth noting that to obtain this result we do not rely on generating function techniques. Those techniques, on the other hand, are used in Section 5 to determine the asymptotic behaviour of the average width of pyramids of large size.

Theorem 1.2 Given $a \geq 2$, the average width of pyramids of size $m$ is asymptotic to

$$
\sqrt{\frac{\pi}{2} a(a-1) m}
$$

for $m \rightarrow \infty$.

Along the way we also point out a bijective correspondence between right pyramids of size $m$ and $a$-ary trees with $m$ nodes. In Section 6 we conclude with some numerical results and comments concerning the growth rate of the number of general planar LEGOs as a function of size and on the dependence of the exponential growth constant on the size of the building blocks. 


\section{The dimer case}

In this section we assume $a=2$. Hence the pieces in this case can be thought of as dimers.

We first note the following decomposition property.

Lemma 2.1 There is a bijective correspondence between pyramids of size $m \geq 1$ and sequences of pyramids $\left(p_{1}, p_{2}, \ldots, p_{r}\right)$ such that $p_{i}$ is a right 0 -pyramid if $i$ is odd and a left 1-pyramid if $i$ is even, and such that $\left|p_{1}\right|+\ldots+\left|p_{r}\right|=m$.

Proof: If the pyramid $p$ is not a right 0-pyramid there is a lowest piece in $p$ above the interval $]-1,1[$ and this piece is the bottom piece of a unique proper sub-pyramid $p^{\prime}$ and we can write $p=p_{1} \odot p^{\prime}$ where $p_{1}$ is a right 0-pyramid. Here we use the notation of [25] such that, given two heaps $h$ and $h^{\prime}$, the heap $h \odot h^{\prime}$ is obtained by first dropping the pieces of $h$ towards the horizontal axis and then those of $h^{\prime}$. If $p^{\prime}$ is not a left 1-pyramid it contains a unique lowest piece above the interval $] 0,2\left[\right.$ and we have $p^{\prime}=p_{2} \odot p^{\prime \prime}$, where $p_{2}$ is a left 1-pyramid. Repeating the argument the claim follows. See also [6] for a similar decomposition.

Definition 2.2 A finite sequence of 0 's and 1 's will be called $a$ string and by an $(n, m)$-string we mean a string of length $n$ with $m$ 1's. A $(2 m, m)$-string $x_{1} x_{2} \ldots x_{2 m}$ is called positive if

$$
t_{s} \equiv \sum_{u=1}^{s}\left(2 x_{u}-1\right)
$$

is non-negative for all $s=1, \ldots, 2 m$, i.e., the number of 0 's in $x_{1} \ldots x_{s}$ at most equals the number of 1 's in $x_{1} \ldots x_{s}$ for each $s$.

Note that a positive $(2 m, m)$-string necessarily begins with a 1 and ends with a 0 . There is a natural correspondence between strings and nearest neighbouring walks on the integers starting at 0 where each 0 corresponds to a left-step and each 1 to a right-step. Positive strings then correspond to walks on the non-negative integers starting at 0 .

Lemma 2.3 There is a bijective correspondence between positive $(2 m, m)$-strings and right 0 -pyramids.

Proof: Let $p$ be a right 0-pyramid of size $m$. We construct inductively the corresponding positive string $x_{1} \ldots x_{2 m}$ together with a sequence $p^{(1)} \ldots p^{(2 m)}$ of 0 -pyramids that are sub-pyramids of $p$ such that $p^{(2 m)}=p$ as follows.

Let $x_{1}=1$ and $p^{(1)}$ be the bottom piece of $p$. Assume $x_{1} \ldots x_{s}$ and $p^{(1)} \ldots p^{(s)}$ have been constructed. If a piece above the interval $] t_{s}, t_{s}+2\left[\right.$ can be dropped onto $p^{(s)}$ to obtain a sub-pyramid of $p$ we let $p^{(s+1)}$ be that pyramid and set $x_{s+1}=1$. Otherwise, set $p^{(s+1)}=p^{(s)}$ and $x_{s+1}=0$. Here $t_{s}$ is given as in Definition 2.2 and one readily checks that at any stage $t_{s}$ is less than the width of $p^{(s)}$, i.e., the length of the projection of $p^{(s)}$ onto the horizontal axis, and that the size of $p^{(s)}$ equals the number of 1 's in $x_{1} \ldots x_{s}$. Indeed, by construction, any piece that can be dropped onto $p^{(s)}$ to obtain a sub-pyramid of $p$ is above some interval contained in $] 0, t_{s}+2[$, and at any stage we choose the rightmost of those pieces to obtain $p^{(s+1)}$. It follows that the so obtained sequence $x_{1} \ldots x_{2 m}$ after $2 m$ steps is a positive $(2 m, m)$-string since otherwise the number of 1's would be less than $m$ and $t_{2 m}$ would hence be negative, which is not possible. 


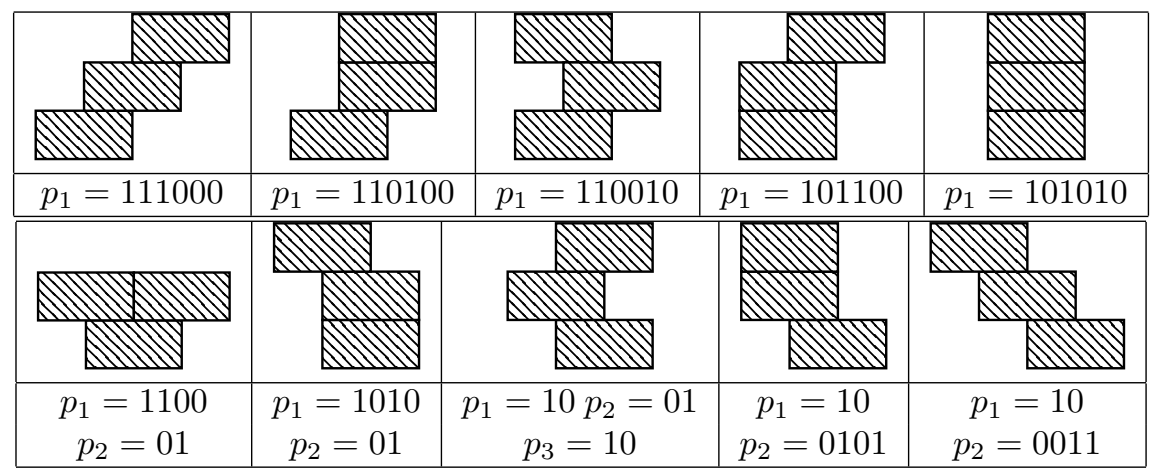

Fig. 1: The ten 2-pyramids of size 3 and their description as $(6,3)$-strings

If $p \neq p^{\prime}$, the corresponding sequences $p^{(1)} \ldots p^{(2 m)}$ and $p^{(1)} \ldots p^{(2 m)}$ will deviate at some minimal step $s, 1<s \leq 2 m$, and it follows that the corresponding strings also deviate at step $s$. On the other hand, any positive $(2 m, m)$-string $x_{1} \ldots x_{2 m}$ can be obtained by the described procedure from the right 0 -pyramid $p$ obtained by successively dropping a piece above those intervals $] t_{s}, t_{s}+2\left[\right.$ for which $x_{s}=1$, with the convention $t_{0}=0$. This concludes the proof.

Lemma 2.4 Any $(2 m, m)$-string $w=x_{1} \ldots x_{2 m}$ starting with $x_{1}=1$ can be written in a unique way by juxtaposition as

$$
w=w_{1} \ldots w_{r}
$$

where $w_{i}$ is a positive 0-string if $i$ is odd, and $w_{i}^{-1}$ is a positive 0 -string if $i$ is even. Here $w_{i}^{-1}$ denotes the string obtained from $w_{i}$ by reversing its order.

Proof: Using the correspondence between strings and nearest neighbouring walks on the integers, we see that the statement amounts to asserting the obvious unique decomposition of a walk starting and ending at 0 into an alternating sequence of walks on the non-negative, respectively the non-positive, integers.

We are now in a position to derive the following result which, in particular, proves Theorem 1.1 in case $a=2$.

Proposition 2.5 There is a bijective correspondence between pyramids of size $m$ and $(2 m, m)$-strings starting with 1 . In particular, the number of pyramids of size $m$ equals $\left(\begin{array}{c}2 m-1 \\ m-1\end{array}\right)$.

Proof: Since, obviously, there is a bijective correspondence between left and right 0-pyramids of given size and since reversal of ordering of a string is injective, the claimed correspondence follows from the preceding three lemmas. The last statement follows by noting that a $(2 m, m)$-string starting with 1 is uniquely determined by the position of the remaining 1's among the remaining $2 m-1$ entries of the string. 


\section{Decomposition of pyramids and strings for $a \geq 3$}

In this and the subsequent section we assume $a$ is fixed and larger than or equal to 3. Accordingly, we extend the notion of positive strings as follows.

Definition 3.1 An $(a m, m)$-string $x_{1} x_{2} \ldots x_{a m}$ is called positive if

$$
t_{s} \equiv \sum_{u=1}^{s}\left(a x_{u}-1\right)
$$

is non-negative for all $s=1, \ldots$, am. Moreover, a string is called negative if the reversed string is positive.

Note that a positive string necessarily begins with 1 and ends with at least $a-1$ consecutive 0 's.

The following two lemmas are simple generalisations of Lemmas 2.1 and 2.3

Lemma 3.2 There is a bijective correspondence between pyramids of size $m \geq 1$ and sequences of pyramids $\left(p_{1}, p_{2}, \ldots, p_{r}\right)$ such that $p_{i}$ is a right $s_{i}$-pyramid if $i$ is odd and a left $s_{i}$-pyramid if $i$ is even, $\left|p_{1}\right|+\ldots+\left|p_{r}\right|=m, s_{1}=0$, and

$$
1 \leq s_{i+1}-s_{i} \leq a-1 \quad \text { if } i \text { is odd } \quad \text { and } \quad 1 \leq s_{i}-s_{i+1} \leq a-1 \text { if } i \text { is even. }
$$

Proof: If the pyramid $p$ is not a right 0-pyramid there is a lowest piece in $p$ above some interval that overlaps the negative real axis, that is an interval $] s_{2}-a, s_{2}\left[\right.$, where $0<s_{2}<a$. This piece is the bottom piece of a unique proper sub-pyramid $p^{\prime}$ and we can write $p=p_{1} \odot p^{\prime}$, where $p_{1}$ is a right 0 pyramid. If $p^{\prime}$ is not a left $s_{2}$-pyramid it contains a unique lowest piece above some interval $] s_{3}, s_{3}+a$, where $s_{2}-a<s_{3}<s_{2}$. This piece is the bottom piece of a proper sub-pyramid $p^{\prime \prime}$ of $p^{\prime}$, and we have $p^{\prime}=p_{2} \odot p^{\prime \prime}$, where $p_{2}$ is a left $s_{2}$-pyramid. The claim follows by repeating the argument a sufficient number of times.

Lemma 3.3 There is a bijective correspondence between right 0-pyramids of pieces of length a and of size $m$ and positive $(a m, m)$-strings.

Proof: The claim follows by a straight-forward generalisation of the proof of Lemma 2.3, the details of which are left to the reader.

The following related correspondence between right pyramids and trees will not be needed in the proof of Theorem 1.1. but may be of some independent interest. Recall that an $n$-ary tree, where $n$ is a fixed positive integer, is a planar rooted tree all of whose vertices have order 1 or $n+1$ and whose root has order 1 . The vertices of order $n+1$ are called nodes.

Proposition 3.4 There is a bijective correspondence between right 0-pyramids of pieces of length a and of size $m$ and a-ary trees with $m$ nodes.

Proof: First, note that there is an obvious bijective correspondence between $(a m, m)$-strings and walks on the integers starting and ending at 0 and consisting of $m$ right-steps, each of length $a-1$, and $(a-1) m$ left-steps, each of length 1 . In fact, $t_{s}$ as given in Definition 3.1 defines the $s^{\prime}$ th site visited by the walk corresponding to a given string. Alternatively, the corresponding walk can be viewed as a path on the 
square lattice $\mathbb{Z}^{2}$ from $(0,0)$ to $(a m, 0)$ with steps $(1, a-1)$ or $(1,-1)$, called up-steps and down-steps, respectively. Positive strings then correspond to paths with vertices on or above the first axis only, and they are called generalised Dyck $(a-1)$-paths [7], [8].

Consider a generalised Dyck $(a-1)$-path $\omega$ and let $\omega^{\prime}$ be the path obtained by removing the first step, which is necessarily an up-step. Thus $\omega^{\prime}$ starts at height $a-1$ and ends at height 0 . Let now $\omega_{1}$ be the part of $\omega^{\prime}$ extending from the first vertex in $\omega^{\prime}$ at height 0 to the final vertex $(0,0)$. Then $\omega_{1}$ is a generalised $(a-1)$-Dyck path (possibly trivial), and $\omega^{\prime}$ equals a path $\omega^{\prime \prime}$ starting at level $a-1$, ending at level 1 and nowhere dropping below level 1 , followed by first a down-step and then by $\omega_{1}$. Next, let $\omega_{2}$ be the part of $\omega^{\prime \prime}$ extending from the first vertex in $\omega^{\prime \prime}$ at height 1 to the final vertex $\left(x_{1}, 1\right)$. Then $\omega_{2}$ is a translated generalised Dyck $(a-1)$-path and the construction may be repeated $a$ times to yield a decomposition of $\omega$ into a sequence $\omega_{a}, \omega_{a-1}, \ldots, \omega_{1}$ of generalised $(a-1)$-Dyck paths (suitably translated and possibly trivial) connected by single down-steps and preceded by an up-step. As a consequence, the number $A_{m}$ of generalised $(a-1)$-Dyck paths with $m$ up-steps satisfies the recursion relation

$$
A_{m}=\sum_{\substack{m_{1}+\ldots, m_{a}=m-1 \\ m_{1}, \ldots, m_{a} \geq 0}} A_{m_{1}} \cdot \ldots \cdot A_{m_{a}}
$$

Rephrased in terms of the generating function

$$
A(t)=\sum_{m=1}^{\infty} A_{m} t^{m}
$$

this relation takes the form

$$
A(t)=t(1+A(t))^{a} .
$$

This identity has been noted previously in [7] (for $a=3$ ). It is well known, and easy to establish, that the generating function for the number of $a$-ary trees as a function of the number of nodes likewise satisfies (2). We conclude that the coefficients are equal and hence, in view of Lemma 3.3, the claimed bijection is established.

Corollary 3.5 The number $A_{m}$ of right pyramids of size $m$ is given by

$$
A_{m}=\frac{1}{(a-1) m+1}\left(\begin{array}{c}
a m \\
m
\end{array}\right)=\frac{(a m) !}{m !((a-1) m+1) !} \quad m \geq 1 .
$$

Proof: It is known that the stated expression for $A_{m}$ equals the number of $a$-ary trees with $m$ nodes, see e.g. [22]. Hence the claim follows from Proposition 3.4

Remark 3.6 It is, in fact, quite straight-forward to show directly, by a slight modification of the argument given in the proof of Proposition 1 in [6], that the generating function for the number of right 0-pyramids as a function of size satisfies the identity (2). The argument involving generalized Dyck paths given above exhibits at the same time a proper generalisation of the well-known, and much exploited, correspondence between binary trees and standard Dyck paths [18]. In turn, it is a particular instance of a correspondence between so-called Lukasiewicz paths and certain classes of trees; see e.g. [12] section VII.8]. 
In order to continue our efforts to establish a decomposition result analogous to Lemma 2.4 some additional notation will be needed. We shall find it convenient to use the language of walks instead of strings in the following. Hence all walks subsequently will be assumed to have right-steps of length $a-1$ and left-steps of length 1 . A generic walk starting at $i \in \mathbb{Z}$ and ending at $j \in \mathbb{Z}$ will be denoted by $S_{i j}(m), S_{i j}^{\prime}\left(m^{\prime}\right)$ etc. For $i=0$ the walk corresponding to an $(n, m)$-string is obtained by letting $t_{s}$ given as in Definition 3.1 be its $s^{\prime}$ th site.

Given two walks $S_{i j}^{\prime}\left(m^{\prime}\right)$ and $S_{j k}^{\prime \prime}\left(m^{\prime \prime}\right)$, the walk obtained by by first traversing $S_{i j}^{\prime}\left(m^{\prime}\right)$ and then $S_{k j}^{\prime \prime}\left(m^{\prime \prime}\right)$ will be called the walk obtained by composing $S_{i j}^{\prime}\left(m^{\prime}\right)$ and $S_{j k}^{\prime \prime}\left(m^{\prime \prime}\right)$ and will be denoted by $S_{i j}^{\prime}\left(m^{\prime}\right) S_{j k}^{\prime \prime}\left(m^{\prime \prime}\right)$. Thus, composition of walks corresponds to juxtaposition of the corresponding strings.

Evidently, an $(a m, m)$-string is positive if and only if the corresponding walk takes place on the nonnegative integers. Generally, we shall call a walk $S_{i j}(m)$ positive if $j \geq i$ and the walk $S_{i i}(m)$ obtained by adding $j-i$ left-steps at the end is a translate (by $i$ ) of a walk on the non-negative integers. Positive walks will be denoted by $P_{i j}(m), P_{i j}^{\prime}\left(m^{\prime}\right)$ etc.

Given a walk $S_{i j}(m)$, its inverse walk $S_{j(2 j-i)}^{-1}(m)$ is defined as the walk obtained by reflecting $S_{i j}(m)$ in the point $j$ and reversing its direction of traversal. If $i=j=0$ this corresponds to reversing the order of the corresponding string. A walk is called negative if its inverse is a positive walk. Generic negative walks will be denoted by $N_{i j}(m), N_{i j}^{\prime}\left(m^{\prime}\right)$ etc. Note that positive walks begin with a right-step whereas negative walks end with a right-step.

For $0 \leq j<i \leq a-2$ we denote by $T_{i j}$ the straight walk from $i$ to $j$ consisting of $i-j$ left-steps and, for $0 \leq j<k \leq a-2$, we define

$$
S_{i j}(m)=S_{i k}^{\prime}(m) T_{k j} \Leftrightarrow S_{i k}^{\prime}(m)=S_{i j}(m) U_{j k}
$$

that is the last equation means that $S_{i j}(m)$ contains at least $k-j$ consecutive left-steps at the end, and $S_{i k}^{\prime}(m)$ is obtained from $S_{i j}(m)$ by deleting its last $k-j$ steps. In particular, we note that any walk $P_{i i}(m)$ necessarily ends with at least $a-1$ left-steps such that $P_{i i}(m) U_{i k}$ is well-defined, and ends with a left-step, for $0 \leq i<k \leq a-2$.

Definition 3.7 Consider a multiple composition of walks of the types $P_{i i}(m), N_{i i}(m), 0 \leq i \leq a-2$, and $T_{i j}, 0 \leq j<i \leq a-2$, and with possible insertions of terms $U_{i k}, 0 \leq i<k \leq a-2$. By dropping the endpoint indices $i, j, k$ and the step numbers $m$ in the composition we obtain a word in the alphabet $P, N, T, U$. The composition is called admissible if only neighbouring pairs of letters of the form

$$
P N, N P, P T, T P, N T, T N, P U, U N
$$

occur in the corresponding word.

Figure 2 illustrates how a walk can be thought of as a path in $\mathbb{Z}^{2}$, replacing each right-step by an up-step $(1, a-1)$ and each left-step by a down-step $(1,-1)-$ in the case illustrated, $a=6$. It also illustrates how an admissible composition of a walk $S_{0 j}(m)$, where $0 \leq j \leq a-2$, can be found based only on the properties of the path in the horizontal band shown. We are now in a position to formulate the desired decomposition result for walks. The proof is a rather simple matter of book-keeping and is deferred to Appendix A

Lemma 3.8 Any walk $S_{0 j}(m)$, where $0 \leq j \leq a-2$, can be written in a unique way as an admissible composition. 


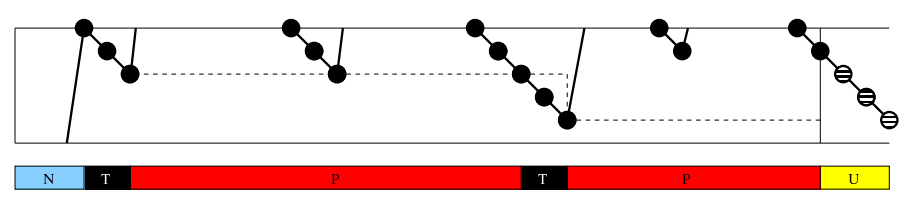

Fig. 2: Walk with decomposition

Corollary 3.9 There is a bijective correspondence between walks of length am starting at 0 with a rightstep and ending at 0 , and admissible compositions with initial term $P_{0 j_{1}}\left(m_{1}\right), 0 \leq j_{1} \leq a-2, m_{1} \geq 1$, and final term $P_{00}\left(m_{r}\right), N_{00}\left(m_{r}\right), m_{r} \geq 1$, or $T_{j_{r} 0}, 1 \leq j_{r} \leq a-2$, where $m_{1}+\ldots+m_{r}=m$.

\section{Proof of Theorem 1.1}

The main purpose of this section is to give a proof of Theorem 1.1 by exploiting the decomposition results of the preceding section.

For $m \geq 1$, we let $A_{m}$ denote the number of positive walks $P_{i i}(m)$, which obviously is independent of $i \in \mathbb{Z}$ and also equals the number of negative walks $N_{i i}(m)$. Moreover, $A_{m}$ also equals the number of right, respectively left, $s$-pyramids of size $m$ as a consequence of Lemma 3.3 .

By Lemma 3.2 it follows that the number $B_{m}$ of all 0-pyramids of size $m$ can be written as

$$
B_{m}=\sum_{r \geq 1} \sum_{m_{1}+\ldots+m_{r}=m}(a-1)^{r-1} A_{m_{1}} \ldots A_{m_{r}},
$$

since the number of possible choices of the sequence $\left(s_{1}, \ldots, s_{r}\right)$ in Lemma 3.2 is $(a-1)^{r-1}$.

On the other hand, it follows from Corollary 3.9 that the number of walks of length am starting at 0 with a right-step and ending at 0 can be written in the form

$$
\sum_{r \geq 1} \sum_{m_{1}+\ldots+m_{r}=m} a_{r} A_{m_{1}} \ldots A_{m_{r}}
$$

where $r$ denotes the total number of $P$ - and $N$-terms, with sizes $m_{1}, \ldots, m_{r} \geq 1$, in a composition and the factor $a_{r}$ counts the number of admissible compositions subject to the boundary conditions specified in Corollary 3.9 for fixed $r$ and $m_{1}, \ldots, m_{r}$. As indicated, this number only depends on $r$. Of course, the total number of walks of length $a m$ starting at 0 with a right-step and ending at 0 equals $\left(\begin{array}{c}a m-1 \\ m-1\end{array}\right)$. Thus the proof of Theorem 1.1 will be completed by proving the following lemma.

Lemma 4.1 For every $a \geq 3$ we have

$$
a_{r}=(a-1)^{r-1}, \quad r \geq 1 .
$$

Proof: We use standard matrix techniques. Set $b:=a-1 \geq 2$ in the following and define the $2 b \times 2 b$ matrices $\mathcal{E}, \mathcal{T}$ and $\mathcal{U}$ by

$$
\mathcal{E}=\mathbf{E} \otimes\left(\begin{array}{ll}
0 & 1 \\
1 & 0
\end{array}\right), \quad \mathcal{T}=\mathbf{T} \otimes\left(\begin{array}{ll}
1 & 1 \\
1 & 1
\end{array}\right), \quad \mathcal{U}=\mathbf{U} \otimes\left(\begin{array}{ll}
0 & 1 \\
0 & 0
\end{array}\right),
$$


where $\mathbf{E}$ is the $b \times b$ unit matrix, $\mathbf{T}$ is the lower triangular $n \times n$-matrix with 1 's below the diagonal and 0's elsewhere, and $\mathbf{U}$ is the transpose of $\mathbf{T}$. We label the rows and columns of the first factor in the tensor products by $i, j \in\{0,1, \ldots, a-2\}$ and those of the second factor by $R, S \in\{P, N\}$. Moreover, we shall use the ordering $0 P, 0 N, 1 P, 1 N, \ldots,(a-2) P,(a-2) N$ of double indices, thus implying the standard identification of the tensor product of a $b \times b$-matrix and a $2 \times 2$-matrix with a $2 b \times 2 b$-matrix.

By construction, $\mathcal{E}_{i R, j S}=1$ if and only if $R_{i i}(m) S_{j j}\left(m^{\prime}\right)$ can occur in an admissible composition, i.e., if $i=j$ and $R \neq S$ according to Definition 3.7. Similarly, $\mathcal{T}_{i R, j S}=1$ if and only if $R_{i i}(m) T_{i j} S_{j j}\left(m^{\prime}\right)$ is allowed, and $\mathcal{U}_{i R, j S}=1$ if and only if $R_{i i}(m) U_{i j} S_{j j}\left(m^{\prime}\right)$ is allowed. Viewing an admissible composition as a chain of links of one of the forms $R_{i i}(m) S_{j j}\left(m^{\prime}\right), R_{i i}(m) T_{i j} S_{j j}\left(m^{\prime}\right)$ or $R_{i i}(m) U_{i j} S_{j j}\left(m^{\prime}\right)$, a chain of $r-1$ links contains a total of $r P$ - and $N$-terms. Expanding the power $(\mathcal{E}+\mathcal{T}+\mathcal{U})^{r-1}$ we hence get

$$
a_{r}=\left\{(\mathcal{E}+\mathcal{T}+\mathcal{U})^{r-1}\right\}_{0 P, 0 P}+\left\{(\mathcal{E}+\mathcal{T}+\mathcal{U})^{r-1}\right\}_{0 P, 0 N}+\sum_{j, S}\left\{(\mathcal{E}+\mathcal{T}+\mathcal{U})^{r-1}\right\}_{0 P, j S} \mathbf{T}_{j 0}
$$

where the three terms correspond to the three possible types of final terms in the compositions specified in Corollary 3.9. Since $\mathbf{T}_{j 0}=1$ if $1 \leq j \leq a-2$ and $\mathbf{T}_{00}=0$, the result can be rewritten as

$$
a_{r}=\left(\begin{array}{lllll}
1 & 0 & 0 & \ldots & 0
\end{array}\right)(\mathcal{E}+\mathcal{T}+\mathcal{U})^{r-1}\left(\begin{array}{c}
1 \\
1 \\
\vdots \\
1
\end{array}\right)
$$

Setting $\mathcal{A}=\mathcal{E}+\mathcal{T}+\mathcal{U}$ one can write $\mathcal{A}=\mathcal{X}+\mathcal{Y}$ where $\mathcal{X}$ is the lower triangular $2 b \times 2 b$-matrix whose matrix elements below the diagonal equal 1 and are otherwise 0 , and $\mathcal{B}$ is the upper triangular matrix with matrix elements equal to 1 in slots with even row and column indices above the diagonal and 0 elsewhere, that is

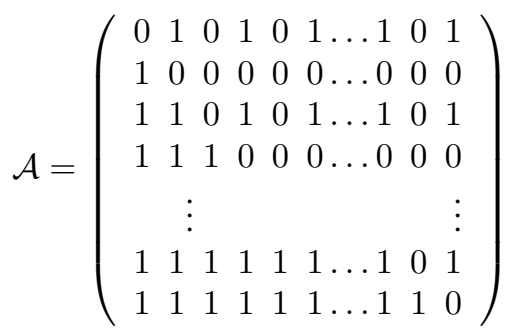

It is now easy to show by induction w.r.t. $b$ that the characteristic polynomial $p_{b}$ of $\mathcal{A}$ is given by

$$
p_{b}(\lambda)=\lambda^{b-1}(\lambda-b)(\lambda+1)^{b} .
$$

Hence the eigenvalues of $\mathcal{A}$ are $0, b$ and -1 , and it is readily seen that they all have eigenvalue multiplicity equal to 1 . We denote by $e$ the eigenvector with eigenvalue $b$ normalised such that its first coordinate is $b$. One finds

$$
e=\left(b, 1, b \zeta, 2 \zeta, b \zeta^{2}, 3 \zeta^{2}, \ldots, b \zeta^{b-2},(b-1) \zeta^{b-2}, b \zeta^{b-1}, b \zeta^{b-1}\right)
$$

where

$$
\zeta=1+b^{-1}
$$


Next, define

$$
f_{i}:=(0, \ldots, 0,-i, 1,1, \ldots, 1), \quad 1 \leq i \leq b-1,
$$

where $-i$ is the $2(b-i)$ 'th coordinate (such that the number of 1 's is $2 i$ ). Then $f_{1}$ belongs to the kernel of $\mathcal{A}$ and one finds by direct computation that

$$
\mathcal{A} f_{i}=f_{1}+f_{2}+\ldots+f_{i-1}, \quad 2 \leq i \leq b-1 .
$$

It follows that the kernel of $\mathcal{A}^{b-1}$ is spanned by the vectors $f_{1}, \ldots, f_{b-1}$. By a straight-forward calculation one finds

$$
(1,1,1, \ldots 1)=b^{-1}\left(e-\sum_{i=1}^{b-1} \zeta^{b-1-i} f_{i}\right) .
$$

From this we conclude that

$$
\begin{aligned}
& a_{r}=b^{-1}\left(\begin{array}{lllll}
1 & 0 & 0 & \ldots 0
\end{array}\right) \mathcal{A}^{r-1}\left(e-\sum_{i=1}^{b-1} \zeta^{b-1-i} f_{i}\right) \\
& =b^{-1}\left(\begin{array}{lllll}
1 & 0 & 0 & \ldots 0
\end{array}\right) \mathcal{A}^{r-1} e \\
& =b^{-1} b^{r-1}\left(\begin{array}{lllll}
1 & 0 & 0 & \ldots & 0
\end{array}\right) e \\
& =b^{r-1} \quad \text { for } r \geq 1 \text {, }
\end{aligned}
$$

where, in the second step, we have used (6) and (7) and the fact that $f_{i}$ has vanishing first coordinate for all $i=1, \ldots, b-1$.

Recalling the definition of $b$ the lemma is proven.

\section{Asymptotic behaviour}

As previously mentioned, the method used in this section to determine the number $B_{m}$ of pyramids of size $m$ as given by Theorem 1.1 did not require knowing the number $A_{m}$ of right-pyramids of size $m$, as given by Corollary 3.5. For $a=2$ the use of generating function techniques as in [6] proceeds by first determining the generating function $A(t)$ for the $A_{m}$ and then using a simple algebraic relation between this function and the generating function

$$
B(t)=\sum_{m=1}^{\infty} B_{m} t^{m}
$$

for the $B_{m}$. For general $a \geq 2$, this relation is a special case of eq. 10 below and takes the form

$$
B(t)=\frac{A(t)}{1-(a-1) A(t)},
$$

For $a=2$ the quadratic relation (2) satisfied by $A(t)$ has a simple solution which easily yields the $B_{m}$ in closed form when inserted into 8 . For $a>2$ such a procedure is less evident. 
Here we shall apply generating function techniques to determine the asymptotic behaviour of the average width of pyramids of large size as given by Theorem 1.2

Proof of Theorem 1.2

First note that the asymptotic behaviour of

$$
B_{m}=\left(\begin{array}{c}
a m-1 \\
m-1
\end{array}\right)
$$

is readily obtained from Stirling's formula and is given by

$$
B_{m} \sim \frac{1}{\sqrt{2 \pi a(a-1) m}}\left(\frac{a^{a}}{(a-1)^{a-1}}\right)^{m}, \quad m \rightarrow \infty .
$$

Next, let $B_{m, n}$ denote the number of pyramids of size $m$ and left width $n$, where the left width of a pyramid $p$ with bottom piece covering the interval $] 0, a[$ equals $n$ if the leftmost interval covered by a piece in $p$ is $]-n, a-n[$, and let

$$
B(t, v)=\sum_{n \geq 0, m \geq 1} B_{n, m} t^{m} v^{n}
$$

be the corresponding generating function. Now recall the decomposition, in the proof of Lemma 3.2, of a pyramid $p$ into a right 0 -pyramid $p_{1}$ and an arbitrary pyramid $p^{\prime}$ with bottom piece covering the interval ]$s_{2}-a, s_{2}\left[\right.$, where $1 \leq s_{2} \leq a-1$, or $p^{\prime}$ may be empty. This is seen to imply the relation

$$
B(t, v)=A(t)\left(1+\left(v+v^{2}+\ldots+v^{a-1}\right) B(t, v)\right)
$$

between $A(t)$ and $B(t, v)$, that is

$$
B(t, v)=\frac{A(t)}{1-\left(v+v^{2}+\ldots+v^{a-1}\right) A(t)} .
$$

In particular, the relation 8 is obtained for $v=1$.

Moreover, differentiating eq. (10) with respect to $v$ and setting $v=1$ we get

$$
C(t)=\frac{1}{2} a(a-1) B(t)^{2} .
$$

where $C(t)$ is the generating function with coefficients

$$
C_{m}=\sum_{n \geq 0} n B_{m, n} .
$$

The asymptotic behaviour of $C_{m}$ can now be obtained by standard singularity analysis [12]. Indeed, from the polynomial equation (2) satisfied by $A(t)$ we conclude that the singularity of $A(t)$ closest to the origin is at

$$
t_{0}=\frac{(a-1)^{a-1}}{a^{a}}
$$


and that

$$
A\left(t_{0}\right)-A(t) \sim c_{0}\left(1-\frac{t}{t_{0}}\right)^{\frac{1}{2}}, \quad t \rightarrow t_{0}
$$

where

$$
c_{0}=(a-1)^{-2} \sqrt{2 a(a-1)} \text { and } A\left(t_{0}\right)=(a-1)^{-1} .
$$

Using $(8)$ and $(11]$ one obtains

$$
C(t) \sim \frac{1}{4}\left(1-\frac{t}{t_{0}}\right)^{-1}, \quad t \rightarrow t_{0},
$$

and hence

$$
C_{m} \sim \frac{1}{4} t_{0}^{-m}=\frac{1}{4}\left(\frac{a^{a}}{(a-1)^{a-1}}\right)^{m}, \quad m \rightarrow \infty .
$$

For the average left width with respect to the uniform distribution of pyramids of size $m$ we conclude from (9) and (12) that

$$
\frac{C_{m}}{B_{m}} \sim \frac{1}{4} \sqrt{2 \pi a(a-1) m}, \quad m \rightarrow \infty .
$$

This finishes the proof since the average width equals twice the average left width plus $a$.

Remark 5.1 For $a=2$ the square root behaviour of the width was obtained in [6], but the multiplicative constant given there is not correct.

\section{Concluding remarks}

As noted in the introduction the pyramids under consideration in this paper may be considered as special planar LEGO structures built from $1 \times a$ LEGO pieces. More specifically, consider the number $L_{m}^{a}$ of contiguous LEGO structures made out of $m 1 \times a$ pieces which are "flat" in the sense that all pieces are contained in the same vertical plane, and such that there is a unique piece in the lowest level of the structure. Pictorially speaking, the difference between that case and the one studied here is that pieces are allowed to hang underneath other pieces from the second level of the structure and upwards. These numbers turn out to be hard to compute; in [2] some of them have been calculated for $1 \leq a \leq 8$ and are reproduced in The Online Encyclopaedia of Integer Sequences [21].

In [11] we have shown, using standard concatenation arguments, that the exponential growth rate

$$
g_{a}=\lim _{m \rightarrow \infty} \frac{\ln L_{m}^{a}}{m}
$$

is well defined and finite (and that this, in fact, holds also for more general non-planar classes of LEGO structures). Obviously, the asymptotic relation 9 ) gives

$$
h_{a} \geq \frac{a^{a}}{(a-1)^{a-1}} \sim e^{-1}(a-1)
$$




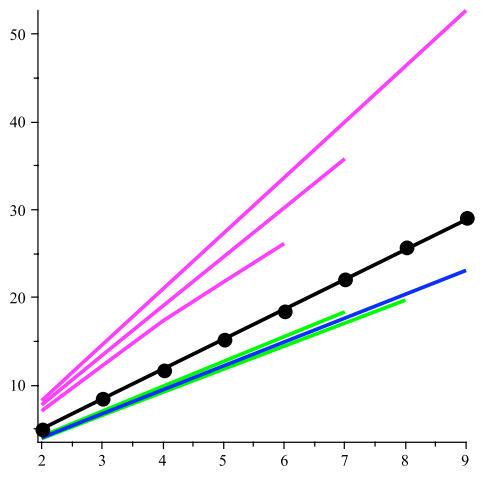

Fig. 3: Black: our conjectural formula for $h_{a}$ and the estimated values. Magenta: Upper bounds based on the Klarner-Rivest method (levels 1, 2 and 3). Blue: Lower bound from pyramids. Green: Lower bounds from counting fat buildings (levels 6 and 8).

for large $a$, where we have set $h_{a}=e^{g_{a}}$. This lower bound appears to be rather tight; it is, indeed, at present our best lower bound for general $a$. It may be improved by known techniques for fixed and relatively small values of $a$. For instance, for $a=2$ one can appeal to the enumeration of multi-pyramids in [6] to get $h_{2} \geq 9 / 2$. By adapting the method of [11] to this setting we can improve this lower estimate further to $h_{2} \geq 4.607$ by computing the number $c_{m}$ of "fat" structures up to level 16 and proving $c_{m+2} \geq$ $5 c_{m}$ for all $m$.

Upper bounds on $h_{a}$ can be produced by adapting the method of [17] to this setting. Performing an analysis of depth 1 we can prove that, for all $a$, the largest root of

$$
\begin{aligned}
& \left(\frac{1}{4} a^{9}-\frac{4}{5} a^{8}+\frac{21}{8} a^{7}-3 a^{6}+2 a^{5}-\frac{3}{4} a^{4}+\frac{1}{8} a^{3}\right) x^{5} \\
+ & \left(-3 a^{8}+\frac{77}{4} a^{7}-\frac{105}{2} a^{6}+\frac{159}{2} a^{5}-73 a^{4}+\frac{165}{4} a^{3}-\frac{27}{2} a^{2}+2 a\right) x^{4} \\
+ & \left(-\frac{47}{8} a^{7}+27 a^{6}-\frac{195}{4} a^{5}+\frac{85}{2} a^{4}-\frac{135}{8} a^{3}+\frac{3}{2} a^{2}+\frac{1}{2} a\right) x^{3} \\
+ & \left(a^{6}-4 a^{5}+6 a^{4}-4 a^{3}+a^{2}\right) x^{2}
\end{aligned}
$$

is a majorant of $h_{a}$, which in turn shows that

$$
h_{a} \leq 6.356 a-4.375
$$

for large $a$. No closed form upper bound is available for analyses of depth 2 and 3, but majorants are readily computable for $a$ up to 8 as indicated Figure 3 . Again, these appear to be approximately affine for large $a$.

Using the Monte Carlo methods described in [1] and [2] we have produced estimates of $h_{a}$ for $a$ up to 8. Strikingly, our estimates in each case have the form $k_{a} \frac{a^{a}}{(a-1)^{a-1}}$, with $k_{a}$ between 1.238 and 1.264 . This makes it tempting to speculate that

$$
h_{a}=\frac{5 a^{a}}{4(a-1)^{a-1}} .
$$


In particular, it does not seem unlikely that $h_{2}=5$. Our best current estimate, achieved by a least square fitting of a function of the form $A H^{n} n^{C}$ with Monte Carlo estimates for $L_{16}^{2}, \ldots, L_{20}^{2}$ yields $H=5.0012$.

\section{Acknowledgements}

We thank two anonymous referees for valuable suggestions for improvements and corrections.

\section{References}

[1] M. Abrahamsen and S. Eilers, "On the asymptotic enumeration of LEGO structures", to appear in Experimental Math.

[2] M. Abrahamsen and S. Eilers, "Efficient counting of LEGO buildings", technical report.

[3] E. Barcucci, A. Del Lungo, E. Pergola and R. Pinzani, "Directed animals, forests and permutations", Discrete Math. 204 (1999), 103-117.

[4] J. Bétréma and J.-G. Penaud, "Animaux et arbres guingois", Theoret. Comput. Sci. 117 (1993), 67-89.

[5] J. Bétréma and J.-G. Penaud, "Modèles avec particules dures, animaux dirigés et séries en variables partiellement commutatives", arXiv:math.CO/0106210, Technical Report 93-18. LaBRI, Université Bordeaux 1 (1993).

[6] M. Bousquet-Mélou and A. Rechnitzer, "Lattice animals and heaps of dimers", Discrete Math. 258 (2002), 235-274.

[7] N. T. Cameron, "The combinatorics of even trees", Proc. of the 31. Southeastern International Conf. on Combinatorics, Graph theory and Computing, Congr. Numer. 147 (2000), 129-143.

[8] N. T. Cameron, "Random Walks, Trees and Extensions of Riordan Group Techniques", Annual Joint Mathematics Meetings, Baltimore, MD, 2003.

[9] A. R. Conway and A. J. Guttmann, "On two-dimensional percolation”, J. Phys. A: Math. and Gen. 28 (1995), 891-904.

[10] D. Dhar, "Equivalence of the two-dimensional directed-site lattice animal problem to Baxter's hard square lattice gas model", Phys. Rev. Lett. 49 (1982), 959-962.

[11] B. Durhuus and S. Eilers, "On the entropy of LEGO”, arXivmath/0504039 (2005).

[12] P. Flajolet and R. Sedgewick, Analytic Combinatorics, Cambridge University Press, 2009

[13] G. Grimmett, "Percolation", Grundlehren der mathematischen Wissenschaften, Vol. 321, Springer Verlag (1999).

[14] D. Gouyou-Beauchamps and G. Viennot, "Equivalence of the two-dimensional directed animal problem to a one-dimensional path problem", Adv. Appl. Math. 9 (1988), 334-357. 
[15] V. Hakim and J.-P. Nadal, "Exact results for 2D directed animals on a strip of finite width", J. Phys. A: Math. Gen. 9 (1983), L213-L218.

[16] D. A. Klarner, "Some results concerning polyominoes", Fibonacci Quart. 3 (1965), 9-20.

[17] D. A. Klarner and R. L. Rivest, "A procedure for improving the upper bound for the number of $n$-ominoes", Can. Journ. Math. 25 (1973), 585-602.

[18] F. Le Gall, "Random trees and applications", Probability Surveys 2 (2005), 245-311.

[19] J.-G. Penaud, "Une nouvelle bijection pour les animaux dirigés", in: Actes du 22ème séminaire lotharingen de combinatoire, Université de Strassbourg, France, 1989.

[20] L. W. Shapiro, "Directed animals and Motzkin paths", preprint, Howard University, Washington DC (1999).

[21] N. J. A. Sloane, "The Online Encyclopaedia of Integer Sequences": A123768, A123776, A123784, A123792, A123800, A123808, A123816.

[22] R. P. Stanley, Enumerative Combinatorics, Vol 2, Cambridge Studies in Advanced Mathematics 62, Cambridge University Press 1999.

[23] H. N. V. Temperley, "Combinatorial problems suggested by the statistial mechanics of domains and of rubber-like molecules", Phys. Rev. 101 (1956), 1-16.

[24] G. X. Viennot, "Problèmes combinatoires posès par la physique statistique", Asterisque 121-122 (1985), 225-246 (Soc.Math. France).

[25] G. X. Viennot, "Heaps of pieces, I: Basic definitions and combinatorial lemmas", in: G. Labelle and P. Leroux (eds.) Lecture Notes in Mathematics 1234 (1986), 321-350.

[26] D. Zeilberger, “Automated counting of LEGO towers”, J. Difference Equ. 5 (1999), 323-333.

\section{A Proof of Lemma 3.8}

Proof: Let the walk $S_{0 j}(m)$ be given and let us consider the two possible options, depending on the direction of its last step, separately.

i) If $S_{0 j}(m)$ ends with a right-step, any composition of the claimed type must be of the form

$$
S_{0 j}(m)=S_{0 j}^{\prime}\left(m^{\prime}\right) N_{j j}\left(m-m^{\prime}\right),
$$

where $S_{0 j}^{\prime}\left(m^{\prime}\right)$ is either empty or is a composition that ends with a left-step, since walks corresponding to pairs in the list (3) not ending with $N$ must end with a left-step. On the other hand, there evidently exists a unique $S_{0 j}^{\prime}\left(m^{\prime}\right)$ ending with a left step such that the decomposition above holds. This case is illustrated in Figure 4

ii) If $S_{0 j}(m)$ ends with a left-step, any composition of the claimed type must end with a $P_{j j}\left(m^{\prime}\right)$, a $T_{i j}$ or a $U_{i j}$. 


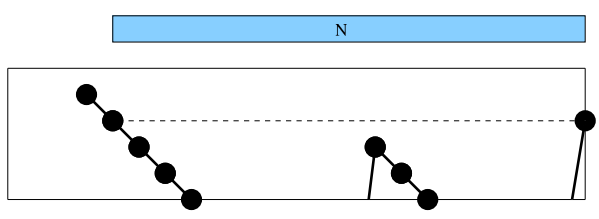

Fig. 4: Decomposition with string ending in $N$

If it ends with a $P_{j j}\left(m^{\prime}\right)$ and is not positive, the composition must have one of the following three forms:

$$
\begin{aligned}
& S_{0 j}(m)=S_{0 j_{1}}^{\prime}\left(m_{0}\right) N_{j_{1} j_{1}}\left(m_{1}\right) T_{j_{1} j_{2}} P_{j_{2} j_{2}}\left(m_{2}\right) T_{j_{2} j_{3}} P_{j_{3} j_{3}}\left(m_{3}\right) \ldots T_{j_{r} j} P_{j j}\left(m^{\prime}\right), \quad r \geq 1, \\
& S_{0 j}(m)=S_{0 j_{1}}^{\prime}\left(m_{0}\right) N_{j_{1} j_{1}}\left(m_{1}\right) P_{j_{1} j_{1}}\left(m_{2}\right) T_{j_{1} j_{2}} P_{j_{2} j_{2}}\left(m_{2}\right) T_{j_{2} j_{3}} P_{j_{3} j_{3}}\left(m_{3}\right) \ldots T_{j_{r} j} P_{j j}\left(m^{\prime}\right), \quad r \geq 1, \\
& S_{0 j}(m)=S_{0 j}^{\prime}\left(m_{0}\right) N_{j j}\left(m_{1}\right) P_{j j}\left(m^{\prime}\right),
\end{aligned}
$$

where $S_{0 j}^{\prime}\left(m_{0}\right)$ is either empty or a composition of the claimed form, because $P$ can only be preceded by $T$ or $N$ and $T$ can only be preceded by $P$ or $N$. Setting $j_{1}=j$ in the last case it is seen that in all three cases the last step in $N_{j_{1} j_{1}}\left(m_{1}\right)$ is the last (right) step, call it $\alpha$, in $S_{0 j}(m)$ whose initial point has negative value and whose final point is non-negative, and hence belongs to $\{0,1, \ldots, a-2\}$. In case $S_{0 j}(m)$ is positive we must have $j=0$ and $S_{0 j}(m)=P_{j j}(m)$.

That the $P$-and $T$-terms occurring in these compositions are uniquely determined can be seen as follows. Consider the step $\alpha$ defined above with endpoint $j_{1}$. If the subsequent step is a left-step there exists a $j_{2}<j_{1}$ such that $\alpha$ is followed by $T_{j_{1} j_{2}}$ and then by a right-step. This right-step is the initial step of a unique positive walk $P_{j_{2} j_{2}}\left(m_{2}\right)$ that is followed by a left-step, unless it equals $P_{j j}\left(m^{\prime}\right)$. If not, the argument can then be repeated. If the first step after $\alpha$ is a right-step, it is the initial step of a unique positive walk $P_{j_{2} j_{2}}\left(m_{2}\right)$ that is followed by a left-step, unless it equals $P_{j j}\left(m^{\prime}\right)$. Now continue as previously until all $P$ - and $T$-terms have been determined.

In case the composition ends with a $T_{i j}$ or a $U_{i j}$, essentially the same argument can be applied to establish uniqueness of the factors subsequent to the step $\alpha$ defined above. If $\alpha$ does not exist, i.e., if $S_{0 j}(m)$ is positive, the unique composition of the claimed type must in this case be $S_{0 j}(m)=P_{00}(m) U_{0 j}$. The case of a composition ending in $P U$ is illustrated in Figure 2

To establish existence of the composition for the part of $S_{0 j}(m)$ subsequent to $\alpha$ one can proceed along the same lines just explained concerning uniqueness. Indeed, if $\alpha$ is followed by a left-step there must exist a non-negative $j_{2}<j_{1}$ such that $\alpha$ is followed by $T_{j_{1} j_{2}}$, which is then followed by a right-step. This right-step is the first step of a $P_{j_{2} j_{2}}\left(m_{2}\right)$. Choosing $m_{2}$ maximal, it follows that $P_{j_{2} j_{2}}\left(m_{2}\right)$ is either followed by a left-step, in which case the construction can be repeated, or the end of $P_{j_{2} j_{2}}\left(m_{2}\right)$ coincides with that of $S_{0 j}(m)$, in which case the construction is finished, or the end of $P_{j_{2} j_{2}}\left(m_{2}\right)$ exceeds that of $S_{0 j}(m)$ by $j-j_{2}>0$ left-steps, in which case these are annihilated by inserting $U_{j_{2} j}$ at the end.

The case where $\alpha$ is followed by a right-step is treated in the same way.

Together, i) and ii) prove the assertion of the lemma by induction. 